\title{
Development of de novo diabetes in long-term follow-up after bariatric surgery
}

\begin{abstract}
Introduction: While bariatric surgery leads to significant prevention and improvement of type 2 diabetes, patients may rarely develop diabetes after bariatric surgery. The aim of this study was to determine the incidence and the characteristic of new-onset diabetes after bariatric surgery over a 17-year period at our institution. Methods: Non-diabetic patients who underwent bariatric surgery at a single academic center (1997-2013) and had a postoperative glycated hemoglobin (HbA1c) $\geq 6.5 \%$, fasting blood glucose (FBG) $\geq 126 \mathrm{mg} / \mathrm{dl}$, or positive glucose tolerance test were identified and studied. Results: Out of 2263 non-diabetic patients at the time of bariatric surgery, 11 patients had new-onset diabetes in the median follow-up time of 9 years (interquartile range [IQR], 4-12). Bariatric procedures performed were Rouxen-Y gastric bypass $(n=7)$, adjustable gastric banding $(n=3)$, and sleeve gastrectomy $(n=1)$. The median interval between surgery and diagnosis of diabetes was 6 years (IQR, 2-9). At the last follow-up, the median HbA1c and FBG values were 6.3\% (IQR, 6.1-6.5) and $95 \mathrm{mg} / \mathrm{dl}$ (IQR, 85-122), respectively. Possible etiologic factors leading to diabetes were weight regain to baseline $(n=6,55 \%)$, steroid-induced after renal transplantation $(n=1)$, pancreatic insufficiency after pancreatitis $(n=1)$, and unknown $(n=3)$. Conclusion: De novo diabetes after bariatric surgery is rare with an incidence of $0.4 \%$ based on our cohort. Weight regain was common $(>50 \%)$ in patients who developed new-onset diabetes suggesting recurrent severe obesity as a potential etiologic factor. All patients had good glycemic control $(\mathrm{HbA} 1 \mathrm{c} \leq 7 \%)$ in the long-term postoperative follow-up.
\end{abstract}

Keyword: Bariatric surgery; Obesity; Glucose; Glycated haemoglobin; Type 2 diabetes; Gastric bypass; Sleeve gastrectomy 